\title{
The Driving Control Strategy of Pure Electric Vehicle Based on Fuzzy Self-adaptive PID
}

\author{
Xu Shi-wei ${ }^{\mathrm{a}}$, Lu Jing ${ }^{\mathrm{b}}$ and Zhao Xuan ${ }^{\mathrm{c}}$ \\ School of automobile, Chang'an University, Shaanxi710064, China. \\ axushiweide2008@163.com, b2091803849@qq.com, 'bluesky_xuan@163.com
}

\begin{abstract}
Keywords:Automotive engineering, drive control, fuzzy self-adaptive PID, double closed loop control.
\end{abstract}

\begin{abstract}
For the problem of pure electric vehicle drive control, a speed-current double closed loop control strategywasproposed by using the fuzzy self-adaptive PID theory, and thevehicle simulation model was established in Matlab/Simscape.The performance of the drive control strategy was verified bystarting operation condition and NEDC cycle in the simulation model. Simulation results show that the pure electric vehicle drive control strategy based on the fuzzy adaptive PID can execute the driving instructionsquickly and accurately, and the vehiclecan operate stably without static speed error, so the control strategy can improve the vehicle driving performanceeffectively.
\end{abstract}

\section{Introduction}

Compared with traditional fuel vehicles, electric vehicles have many advantages such as high energy efficiency and less environmental pollution, so they have been widely concerned and researched all over the world.However,the lack of battery energy density and less motor drive system efficiency limit the developmentand popularizationof electric vehicles ${ }^{[1]}$. Nowadays PID control strategy is adopted in traditional electric vehicle driving system, which has simple structure and high reliability.But PID control strategy is difficult to meet the requirements when road conditions changingbecause PID parameters need to be frequently updated in real time which will bring poor robustness and big overshoot. For these disadvantages, the speed-current double closed loop control strategy is adopted to design the pure electric vehicle drive control system,so as to ensure constant current feedback control of the drive system, and make the speed without static error and regulate quickly when driving resistance changes suddenly.

\section{Speed-current double closed loop control system for pure electric vehicle}

2.1 Double closed loop control strategy for pure electric vehicle. Because the driving process may encounter many complex road conditions, good drive control strategy should be able to take full advantage of the performance of the drive motor, adjust the motorcurrent and output torquereasonably $^{[2]}$. This paper uses the fuzzy self-adaptive PID control strategy to control the speed of pure electric vehicle and the drive motor current in order to meet vehicle driving demands. The speed-current double closed loop control system is shown in figure 1:

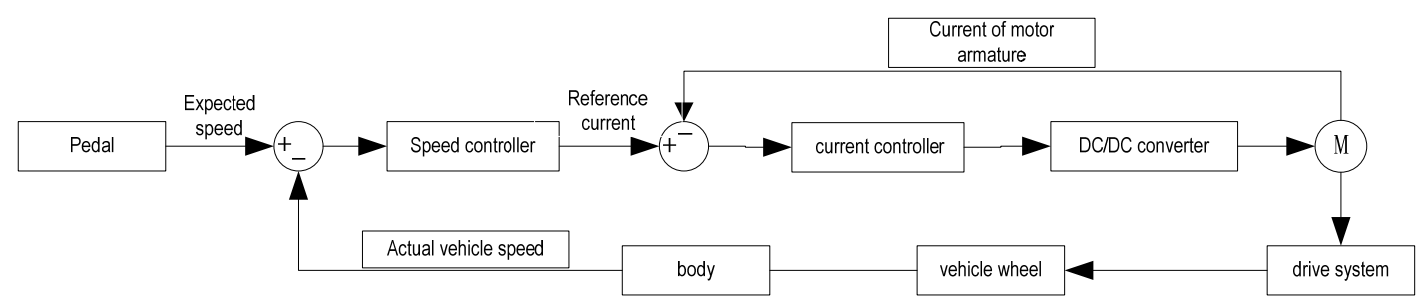

Fig. 1 The speed-current double closed loop control system of pure electric vehicle

In the double closed loop control system, accelerator pedal and brake pedal were controlled based on the vehicle operation environment.According to pedal actions, the expected speed could 
be obtained. The speed regulator of the pure electric vehicle calculated the theoretical armature current of driving motor according to the vehicle speed deviation value, which is obtained by comparing the actual speed feedback signal with the theoretical demand speed. The current deviation value was obtained by comparing the actual drive motor current feedback signal with the theoretical current.Then according to the deviation, the current regulator outputsthe corresponding PWM signal and adjust the power converter on-off time to achieve the vehicle speed control ${ }^{[3]}$.

2.2 Design of fuzzy self-adaptive PID controller based on speed-currentdouble closed loop control system. Compared with the traditional PID control strategy, the fuzzy controller has the advantages such asstrong anti-interference ability, good robustness and strong adaptability.But for complex problems, fuzzy controller will have the disadvantages such as lower accuracy and oscillation when error tends to zero ${ }^{[4,5]}$.In view of above problems, the fuzzy self-adaptive PID control strategyis obtained by combiningthe PID control and fuzzy control, which makes the drive system not only has the advantage of non steady state error of PID control, but also has the advantages such as strong adaptabilityand good robustness characteristics.In this paper, the deviation value and deviation change rate between the actual speed and the theoretical speed are used as the input arguements of the fuzzy self-adaptive PID control strategy. Then the PID parameters are adjusted in real time by using the fuzzy control rules. The structure of fuzzy self-adaptive PID controller is shown in figure 2:

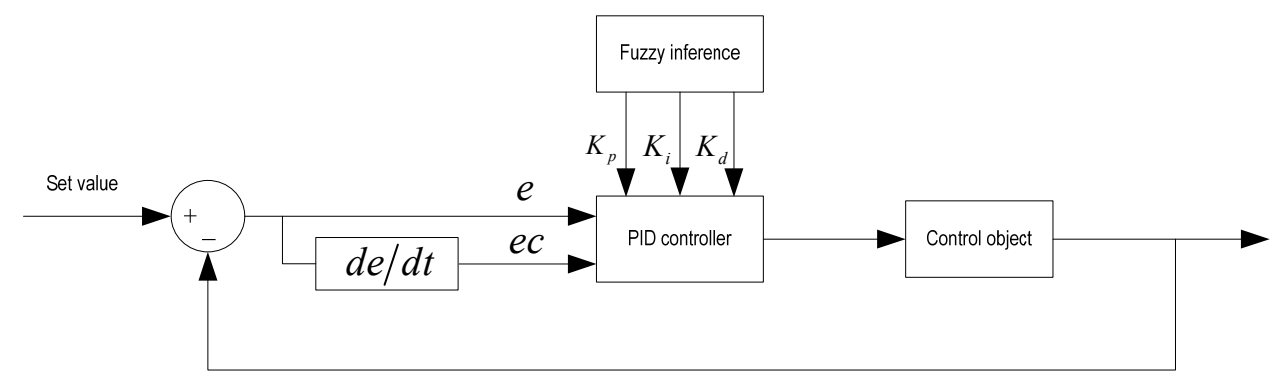

Fig. 2 Structure of the fuzzy self-adaptive PID controller

As shown in Figure 2, the deviation value eand the deviation change rateecareused as inputs arguments of the controller. The ratio coefficient $K_{p}$, the integral coefficient $K_{i}$, and the differential coefficient $K_{d}$ areconducted as the controller's outputs arguments. The PID parameters are adjusted on-line by the controller, so that the system has good static and dynamic characteristics. The fuzzy subsetsof $e$ and $e c$ are divided into seven partitions: NB, NM, NS, ZO, PS, PM, PB.Ultimately, the outputsarguments formulaof fuzzy self-adaptive PID controller were obtained as follows:

$$
\left\{\begin{array}{c}
K_{p}=K_{p 0}+\Delta K_{p} \\
K_{i}=K_{i 0}+\Delta K_{i} \\
K_{d}=K_{d 0}+\Delta K_{d}
\end{array}\right.
$$

In the formula, $K_{p 0}$ is the initial value of the ratio coefficient, $K_{i 0}$ is the initial value of the integral coefficient, $K_{d 0}$ is the initial value of the differential coefficient, $\Delta K_{p}$ is the variation of the proportion coefficient, $\Delta K_{i}$ is the variation of the integral coefficient, and $\Delta K_{d}$ is the variation of the differential coefficient. Thefuzzy membership functionsof $e$ (the deviation value betweenactual speed and the theoretical speed) and $e c$ (the deviation change rate)are shown below: 


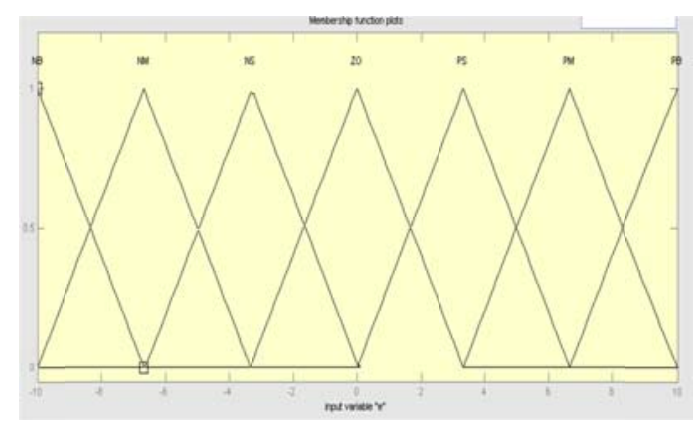

Fig. 3 The fuzzy membership function of the deviation value $e$

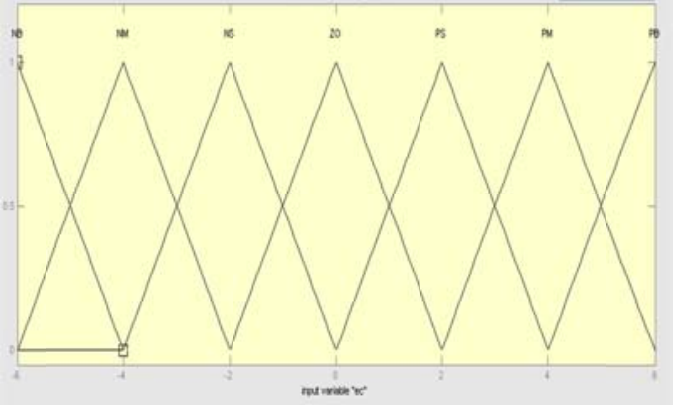

Fig. 4 The fuzzy membership function of the deviation change rate $e c$

Fuzzy control rules are the core of the fuzzy controller, and theyare mainly obtained based on the expert knowledge and technical personnel experiences ${ }^{[5-7]}$. The fuzzy control rulesof the proportion coefficient, integral coefficient and differential coefficient are shown in Table 1:

Table 1 Fuzzy inference rules table

a) Fuzzy control rules table for $K_{p}$

\begin{tabular}{|c|c|c|c|c|c|c|c|}
\hline$e$ & $N B$ & $N M$ & $N S$ & $Z$ & $P S$ & $P M$ & $P B$ \\
\hline$N B$ & $P B$ & $P B$ & $P M$ & $P M$ & $P S$ & $Z$ & $Z$ \\
\hline$N M$ & $P B$ & $P B$ & $P M$ & $P S$ & $P S$ & $Z$ & $N S$ \\
\hline$N S$ & $P M$ & $P M$ & $P M$ & $P S$ & $Z$ & $N S$ & $P S$ \\
\hline$Z$ & $P M$ & $P M$ & $P S$ & $Z$ & $N S$ & $N M$ & $N M$ \\
\hline$P S$ & $P S$ & $P S$ & $Z$ & $N S$ & $N S$ & $N M$ & $N M$ \\
\hline$P M$ & $P S$ & $Z$ & $N S$ & $N M$ & $N M$ & $N M$ & $N B$ \\
\hline$P B$ & $Z$ & $Z$ & $N M$ & $N M$ & $N M$ & $N B$ & $N B$ \\
\hline
\end{tabular}

b) Fuzzy control rules table for Ki

\begin{tabular}{|c|c|c|c|c|c|c|c|}
\hline$e c$ & $N B$ & $N M$ & $N S$ & $Z$ & $P S$ & $P M$ & $P B$ \\
\hline$N B$ & $N B$ & $N B$ & $N M$ & $N M$ & $N S$ & $Z$ & $Z$ \\
\hline$N M$ & $N B$ & $N B$ & $N M$ & $N S$ & $N S$ & $Z$ & $Z$ \\
\hline$N S$ & $N B$ & $N M$ & $N S$ & $N S$ & $Z$ & $P S$ & $P S$ \\
\hline$Z$ & $N M$ & $N M$ & $N S$ & $Z$ & $P S$ & $P M$ & $P M$ \\
\hline$P S$ & $N M$ & $N S$ & $Z$ & $P S$ & $P S$ & $P M$ & $P M$ \\
\hline$P M$ & $Z$ & $Z$ & $P S$ & $P S$ & $P M$ & $P B$ & $P B$ \\
\hline
\end{tabular}




\begin{tabular}{|c|c|c|c|c|c|c|c|}
\hline$P B$ & $Z$ & $Z$ & $P S$ & $P M$ & $P M$ & $P B$ & $P B$ \\
\hline$e$ & $N B$ & $N M$ & $N S$ & $Z$ & $P S$ & $P M$ & $P B$ \\
\hline$e c$ & & & & & & & \\
\hline$N B$ & $P B$ & $N S$ & $N B$ & $N B$ & $N B$ & $N M$ & $P S$ \\
\hline$N M$ & $P S$ & $N S$ & $N B$ & $N M$ & $N M$ & $N S$ & $Z$ \\
\hline$N S$ & $Z$ & $N S$ & $N M$ & $N M$ & $N S$ & $N S$ & $Z$ \\
\hline$Z$ & $Z$ & $N S$ & $N S$ & $N S$ & $N S$ & $N S$ & $Z$ \\
\hline$P S$ & $Z$ & $Z$ & $Z$ & $Z$ & $Z$ & $Z$ & $Z$ \\
\hline$P M$ & $P B$ & $N S$ & $P S$ & $P S$ & $P S$ & $P S$ & $P B$ \\
\hline$P B$ & $P B$ & $P M$ & $P M$ & $P M$ & $P S$ & $P S$ & $P B$ \\
\hline
\end{tabular}

\section{Electric vehicle simulation model based on the speed-current double closed loop control strategy}

According to the speed-current double closed loop control strategy proposed in this paper,the pure electric vehicle model established by Matlab/Simscape is as shown in Figure 5.The system was mainly composed of 6 parts: battery, motor, transmission system, vehicle body, tire and control system. In the system, the speed-current double closed loop control strategy is integrated in the module Controlsystem.First, Controlsystem module got the demand speedfrom the Drive cyclemodule, and used fuzzy self-adaptive PID control strategy to deal with the demandspeed. Then the motor speed control signal and the current control signal were transmitted to the motor and the batteryrespectively to realize the vehicle drive control; After the power was transmitted to the port A of the tire,the translation motion amount could be calculated according to the torque in the port A, and translation motion was passed to the body modulethrough the portH; Vehicle body module mainly calculated the vehicle dynamics, and finally the vehicle speed was concluded according to the displacement amount ofthe tire. In the simulation model, the battery module, DC-DC conversion module and motor module need to be built by SimPowerSystems and SimElectronics.

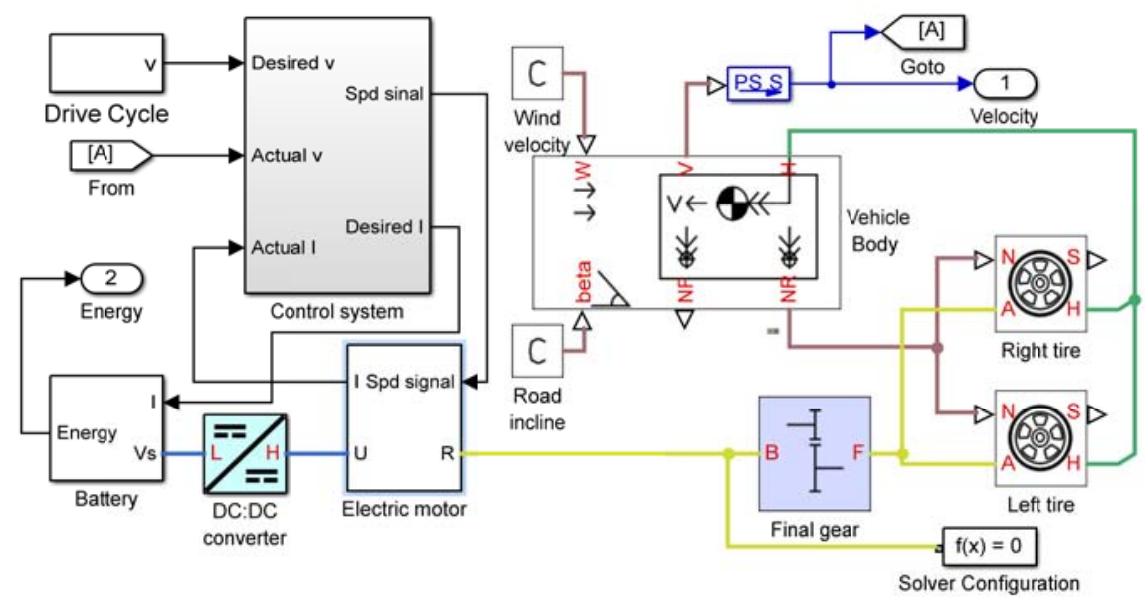

Fig. 5 Electric vehicle simulation model 


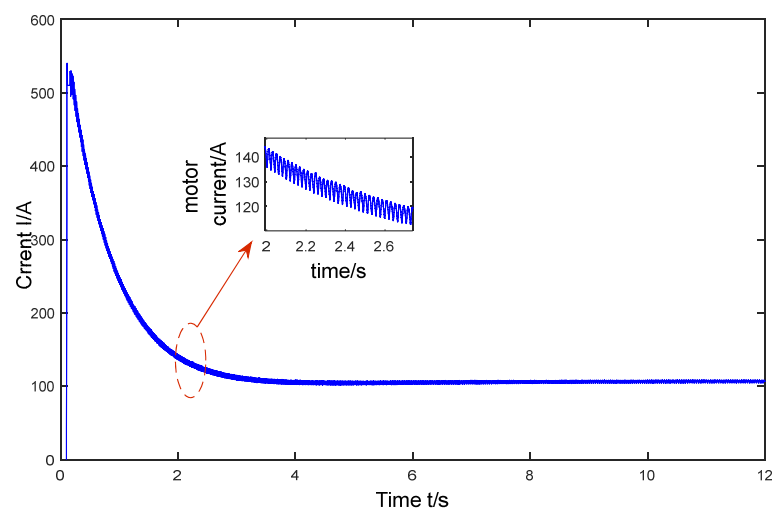

Fig. 6 The motor armature current in startingoperation condition

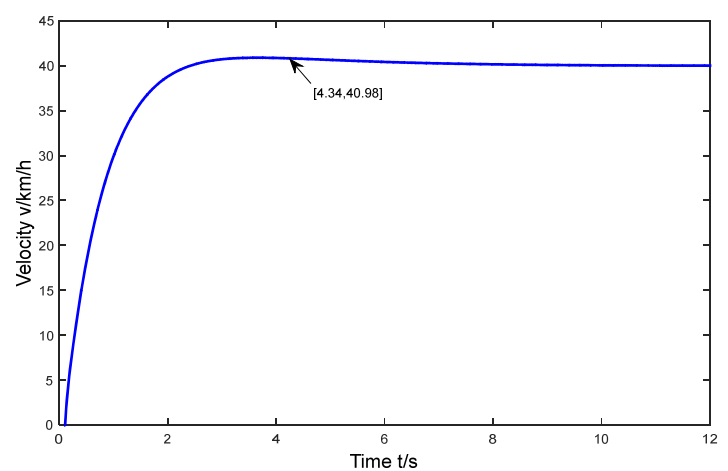

Fig. 7 Pure electric vehicle speedin startingoperation condition

\section{Simulation results and analysis}

In order to verify the performance of the speed-current double closed loop control strategy, thestartingoperation condition and NEDC cycle are selected in the Cycle Driver module, and the simulation results are analyzed as follows.

As shown in Figure 6 and Figure 7, the response time is short and the overshoot is small, so it's much easier to reach stable state when fuzzy self-adaptive PID is used.In $0-0.8 \mathrm{~s}$, the amplitude change of drive motor armature currentis big when the fuzzy self-adaptive PID is adopted, that's because the motor demand torque is quite large at the initial stage, and the armature current needs to produce larger changes to overcome the driving resistance.After $0.8 \mathrm{~s}$, the amplitude change of the armature current and the motor output torque decrease and tend to be stable. At this stage,the fuzzy self-adaptive PID controller solves the shortcomings of less accuracy and oscillations which would be confronted in fuzzy controller, so it is helpful to reach a steady state for drive system. 


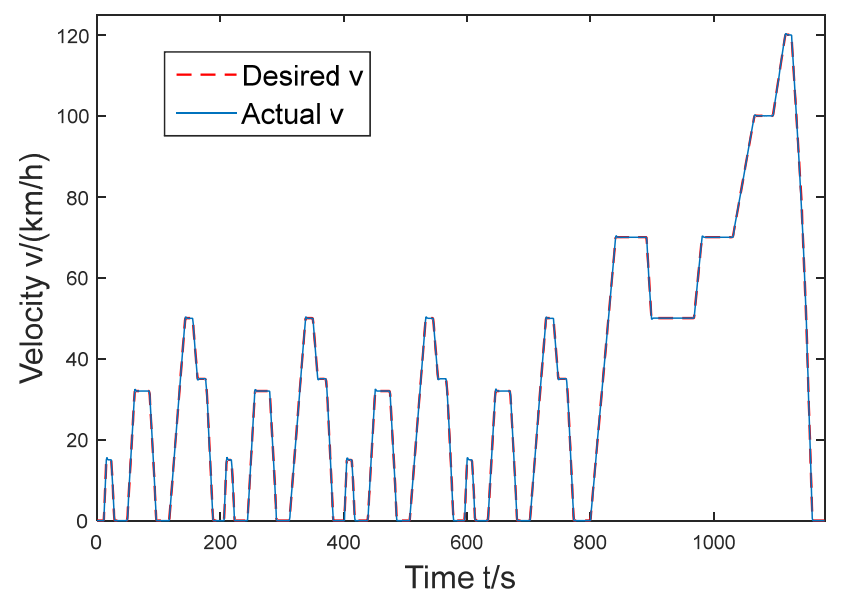

Fig. 8 Speed trace of pure electric vehicle in NEDC cycle

As indicated in Fig. 8, electric vehicle operates stably in NEDC conditions by using the fuzzy self-adaptive PID controller. Except the end of the acceleration stage, the actual speed can accurately follow the desired speed at the reststages. Therefore, the speed-current double closed loop fuzzy self-adaptive PID control strategy proposed in this paper can meet the driving cycle requirements, and effectively improve the vehicle driving performance.

\section{Conclusions}

For the key problem of electric vehicle driving control, after analyzing the traditional PID driving control strategy,the speed-current double closed loop control system with fuzzy adaptive PID control theory was proposed in this paper, and the vehicle simulation model was establishedin Matlab/Simscape. The performance of the drive control strategy was verified bystarting operation condition and NEDC cycle in the simulation model. The simulation results show that the fuzzy self-adaptive PID drive control strategy can achieve the rapid response to vehicle directives andoperate well in complex road cycle. So the control strategy can improve the vehicle driving performanceeffectively.

\section{Acknowledgements}

This work has been supported by NSFC(51507013), Natural science basic research project in Shaanxi Province(2014JQ7269) and Fundamental Research Funds for the Central Universities (310822151025, 310822161002).

\section{Reference}

[1]WANG Q, FRANK A A. Plug-in HEVwith CVT: Configuration, Control, andIts Concurrent Multi-objective Optimization by Evolutionary Algorithm, J. International Journal of Automotive Technology, 15(2014)103-115.

[2]TATE E D, HARPSTER Michael O, SAVAGIAN Peter J. The electrification of the automobile: From Conventional Hybrid, to Plug-in Hybrids, to Extended-Range Electric Vehicles,J. SAE International Journal of Passenger Cars-electronic and Electrical Systems, 1(2008) 156-166.

[3] Li Yong, Ma Fei, KAZERANI Mehrdad. Research on the Control Strategy for the Traction Motor on the Test Bench of Vehicular Energy Storage System,J.Proceedings of the CSEE , 21(2014)3481-3487. 
[4]Song Shu, Gong Jianguo, Lin Wei, Wang Guiping. Modeling and Simulation of Space Vector Control System for Pure Electric Vehicle Driven by Permanent Magnet Synchronous Motor,J. Journal of Wuhan University of Technology,4(2012)118-122.

[5] Tang Dong-sheng, Wu Guang-qiang, Dong Hua-lin. Fuzzy-PID Vector Control for Electric Vehicle, J. Automotive Engineering, 1(2003)61-64.

[6] Zhao Xuan. Study on Control Strategy for Power-train of Pure Electric Bus, D. China, Xi'an, Chang'an University.

[7] Lin Hui. Research on Composite ABS Control Strategy of Fuzzy Self-adjusting PID for Electric-wheel Vehicle, D. China, Changchun, Jilin University. 